\title{
Comparative results of conventional and eversion carotid endarterectomy
}

\author{
Jae Hoon Lee, Bo Yang Suh ${ }^{1}$ \\ Division of Vascular and Endovascular Surgery, Department of Surgery, Catholic University of Daegu School of Medicine, Daegu, \\ ${ }^{1}$ Division of Vascular Surgery, Department of Surgery, Yeungnam University College of Medicine, Daegu, Korea
}

\begin{abstract}
Purpose: Comparative results of conventional carotid endarterectomy (cCEA) and eversion carotid endarterectomy (eCEA) have been reported in many studies. But in Korea, there have been no reports to compare the outcome of the two techniques. Thus, we investigated the results of eCEA compared to cCEA in Yeungnam University Medical Center.

Methods: A total of 120 subjects who underwent CEA were included in this study. Of them, cCEAs were performed in 63 patients and eCEAs were performed in 57 patients. We analyzed the results divided into the early lwithin 30 days after surgery), midterm (from 30 days up to 1 year after surgery) and late lover 1 year after surgery).

Results: Mean age of the patients was $65.9 \pm 7.1$ years in cCEA group and $66.8 \pm 7.7$ years in eCEA group $(P=0.523)$. Carotid shunt frequency was higher in the cCEA group (39.7\% vs. 19.3\%, P = 0.015). There were no statistical differences in the early complications with the exception of a significantly higher risk for new brain lesions in the cCEA group (34.9\% vs. $14.0 \%, P=0.008$ ). The frequency of complication was same between cCEA group and eCEA group in the midterm. Although there was no statistical significance, the frequency of late complications was higher in the cCEA group compared to eCEA group. Mean follow-up duration was $29.4 \pm 23.5$ months.

Conclusion: These data showed that eCEA was an acceptable procedure and had some advantage compared to cCEA in the aspect of the early and late complication.
\end{abstract}

[Ann Surg Treat Res 2014;87(4):192-196]

Key Words: Carotid endarterectomy, Eversion endarterectomy, Carotid stenosis

\section{INTRODUCTION}

Carotid artery stenosis, the narrowing of the lumen of the carotid artery, decreases blood flow to the brain and is known to cause ischemic stroke [1,2]. For treatment of carotid artery stenosis, medical treatment, carotid endarterectomy (CEA) and carotid stenting can be used [3]. Among these therapies, CEA was reported for the first time in 1954 [4]. In the early 1980s, studies regarding CEA showed negative results such as high post operation morbidity [5,6]. Since then, several large multicenter trials verified the safety and durability of CEA [7-10] and CEA is widely accepted as a standard treatment for symptomatic and asymptomatic significant carotid stenosis [11]. There are two main surgical techniques in CEA, conventional carotid endarterectomy (CCEA) and eversion carotid endarterectomy (eCEA). The first includes a standard longitudinal carotid arteriotomy with or without patch angioplasty. The second is done in order of oblique transection, eversion of internal carotid artery (ICA) and reimplantation of the latter into the common carotid artery. eCEA was initially described by De Bakey [12] and later generalized by others [13-15].

Although CEA is known to be safe [7-10], CEA has intraoperative and postoperative risks. Complications that are directly associated with the surgical procedure are strokes,
Received February 6, 2014, Revised March 31, 2014,

Accepted April 15, 2014

\section{Corresponding Author: Jae Hoon Lee}

Division of Vascular and Endovascular Surgery, Department of Surgery, Catholic University of Daegu School of Medicine, 33 Duryugongwon-ro

17-gil, Nam-gu, Daegu 705-718, Korea

Tel: +82-53-650-4623, Fax: +82-53-624-7185

E-mail: iami1124@hanmail.net
Copyright (C) 2014, the Korean Surgical Society

(c) Annals of Surgical Treatment and Research is an Open Access Journal. All articles are distributed under the terms of the Creative Commons Attribution NonCommercial License (http://creativecommons.org/licenses/by-nc/3.0/) which permits unrestricted non-commercial use, distribution, and reproduction in any medium, provided the original work is properly cited. 
myocardial infarction, death, restenosis and so on [16-18]. There are medical complications that are not directly caused by the procedure, such as myocardial infarctions, other cardiovascular disorders, respiratory complications, and transient confusions [19]. Thus, to find a better surgical technique for treatment of carotid artery stenosis is a very important issue; comparative results CCEA and eCEA have been reported in many studies. In Korea, there have been no reports to compare the outcome of the two techniques. Thus, we investigated the results of eCEA compared to CCEA in Yeungnam University Medical Center.

\section{METHODS}

\section{Study population}

From August 2004 to March 2012, 120 patients underwent CEA by one surgeon in Yeungnam University Medical Center. All of these patients were included this study. Of them, cCEAs were performed in 63 patients and eCEAs were performed in 57 patients. The indication of CEA were symptomatic patients with stenosis over seventy percent according to North American Symptomatic Carotid Endarterectomy Trial (NASCET) criteria and asymptomatic patients were considered when they had a stenosis over eighty percent or over fifty percent if the lesion was ulcerative. The luminal area diameter by CT angiography was used for the assessment of carotid artery stenosis. Symptomatic patients were defined as those who had experienced amaurosis fugax, a transient ischemic attack (TIA), or a stroke in the territory of the ipsilateral carotid artery before entry.

\section{Surgical procedure}

Patients had been given at least 1 antiplatelet agent (aspirin, $100 \mathrm{mg}$ daily; cilostazol, $200 \mathrm{mg}$ daily; or clopidogrel, $75 \mathrm{mg}$ daily) for a minimum of 7 days before the operation. CEA was performed under general anesthesia with intraoperative monitoring. Intraoperative monitoring was done by transcranial doppler (TCD) or electroencephalography. Before the clamping of carotid artery, intravenous heparin (5,000 units) was administered to prevent the occurrence of acute thrombosis. When ICA blood flow was reduced over 50\% after clamping of ICA on TCD or the bones of the skull blocked the transmission of ultrasound, selective shunt (Pruitt-Inahara carotid shunt with T-port, LeMaitre Vascular Inc., Burlington, MA, USA) was placed. Surgical options comprised conventional and eCEA. CCEA is preferred when shunt is needed or the level of lesion is high. But it is not a definite indication. The type of operation is at the discretion of the operator. Intraoperative data including the frequency of shunt, clamping time and operation time were examined.

\section{Patient assessment}

Preoperative and postoperative neurological examinations were investigated by a neurologist. MR imaging (Gyroscan Intera 1.5T, Philips Medical Systems, Best, The Netherlands) was performed in all patients within 1 week after treatment. The protocol included isotropic DWI sequence. MRI images were analyzed by experienced neuroradiologists. Duplex scan (iU22 xMATRIX ultrasound system, Philips Medical Systems, Bothell, USA) was performed on all the patients at 1, 3, 6, and 12 months for the first year and annually thereafter. Restenosis was defined as a reduction over $50 \%$ of the lumen and ICA peak systolic velocity over one hundred and twenty-five centimeter per second on Duplex scan. We analyzed the results divided into early (within 30 days after surgery), midterm (from 30 days up to 1 year after surgery) and late (over 1 year after surgery) groupings. Early complications were examined for the frequency of postoperative stroke/TIA, myocardial infarction, cerebral hyperperfusion syndrome, postoperative intracranial hemorrhage, wound complication, cranial nerve palsy, new brain lesions on diffusion-weighted MRI and mortality. Cerebral hyperperfusion syndrome is defined as ipsilateral headache, hypertension, seizures, and focal neurological deficits. Midterm and late complications included restenosis, stroke and mortality.

\section{Statistical analysis}

Statistical analysis was performed using PASW Statistics ver. 18.0 (SPSS Inc., Chicago, IL, USA). Continuous data are reported as mean \pm standard deviation. Nominal data were reported as number of subjects and percentage of individuals affected. Univariate analyses were performed to compare CCEA with eCEA in aspect of complications. For univariate analysis we used chi-square test, Fisher exact test and Student t-test. A P-value $<0.05$ was considered to be statistically significant.

\section{RESULTS}

Demographic and clinical characteristics of total patients are presented in Table 1. A total of 120 patients were included this study. Mean age of the patients was $65.9 \pm 7.1$ years in CCEA group and $66.8 \pm 7.7$ years in eCEA group $(P=0.523)$. The percentage of males was $85.7 \%$ in CCEA group and $89.5 \%$ in eCEA group $(\mathrm{P}=0.534)$. Mean diameter of stenosis was $78.5 \pm 13.3$ in cCEA group and $78.9 \pm 11.4$ in eCEA group (P $=0.864)$ in accordance with NASCET criteria. The common comorbidities were hypertension and smoking followed by diabetes, hyperlipidemia and ischemic heart disease.

Table 2 shows intraoperative data. Shunts were used in 36 cases for the prevention of ischemic events. The number of patients using shunts was 25 of 63 (39.7\%) in CCEA group, 11 of 57 (19.3\%) in eCEA group. Carotid shunt frequency was 
Table 1. Patient demographic and clinical data

\begin{tabular}{lccc}
\hline \multicolumn{1}{c}{ Characteristic } & $\begin{array}{c}\text { CCEA } \\
(\mathrm{n}=63)\end{array}$ & $\begin{array}{c}\text { eCEA } \\
(\mathrm{n}=57)\end{array}$ & P-value \\
Sex & & & \\
$\quad$ Male/female & $54 / 9$ & $51 / 6$ & 0.534 \\
Age (yr) & $65.9 \pm 7.1$ & $66.8 \pm 7.7$ & 0.523 \\
Site & & & 0.883 \\
$\quad$ Right/left & $29 / 34$ & $27 / 30$ & \\
NASCET (\%) criteria & $78.5 \pm 13.3$ & $78.9 \pm 11.4$ & 0.864 \\
Level (C-spine) & $3.2 \pm 0.7$ & $3.2 \pm 0.5$ & 0.691 \\
Comorbidities & & & \\
Peripheral artery disease & $1(1.6)$ & $1(1.8)$ & 0.943 \\
Diabetes mellitus & $22(34.9)$ & $20(35.1)$ & 0.985 \\
Hypertension & $50(79.4)$ & $39(68.4)$ & 0.171 \\
Ischemic heart disease & $8(12.7)$ & $9(15.8)$ & 0.628 \\
Smoking & $32(50.8)$ & $26(45.6)$ & 0.571 \\
Hyperlipidemia & $17(27.0)$ & $22(38.6)$ & 0.175 \\
\hline lues are presented as & &
\end{tabular}

Values are presented as mean \pm standard deviation or number (\%).

cCEA, conventional carotid endarterectomy; eCEA, eversion carotid endarterectomy; NASCET, North American Symptomatic Carotid Endarterectomy Trial; C-spine, cervical spine.

Table 2. Intraoperative data

\begin{tabular}{lccc}
\hline \multicolumn{1}{c}{ Variable } & cCEA $(\mathrm{n}=63)$ & eCEA $(\mathrm{n}=57)$ & P-value \\
Shunt & $25(39.7)$ & $11(19.3)$ & $0.015^{*}$ \\
Clamp time (min) & $21.3 \pm 9.7$ & $24.0 \pm 8.7$ & 0.117 \\
Operation time (min) & $143.9 \pm 36.2$ & $139.9 \pm 30.8$ & 0.521 \\
\hline
\end{tabular}

Values are presented as number $(\%)$ or mean \pm standard deviation.

cCEA, conventional carotid endarterectomy; eCEA, eversion carotid endarterectomy.

${ }^{*} \mathrm{P}<0.05$, statistically significant.

higher in the CCEA group $(\mathrm{P}=0.015)$. There were no statistical differences in clamping time $(21.3 \pm 9.7$ vs. $24.0 \pm 8.7, \mathrm{P}=0.117)$ and operating time $(143.9 \pm 36.2$ vs. $139.9 \pm 30.8)$ between CCEA and eCEA.

We examined the early, midterm and late postoperative complications. Table 3 shows the early complications within 30 days after CEA. There were 7 cases of postoperative stroke/ TIA, 4 of $63(6.3 \%)$ in CCEA group and 3 of $57(5.3 \%)$ in eCEA group $(P=0.800)$. There was no postoperative myocardiac infarction and mortality in both groups. Only 1 case $(1.6 \%)$ of cerebral hyperperfusion syndrome was developed in CCEA group. Intracranial hemorrhage occurred in 2 of $63(3.2 \%)$ in CCEA and 1 of $57(1.8 \%)$ in eCEA ( $P=0.619)$. There was no significant difference in wound complication between CCEA and eCEA ( $3.2 \%$ vs. $1.8 \%, P=0.619)$. The incidence of cranial nerve palsy was similar in the CCEA group compared to eCEA group ( $4.8 \%$ vs. $1.8 \%, P=0.359)$. All of these events were treated conservatively without any complication. There were
Table 3. Early ( $\leq 30$ days) postoperative complications

\begin{tabular}{|c|c|c|c|}
\hline Complication & $\begin{array}{c}\text { CCEA } \\
(\mathrm{n}=63)\end{array}$ & $\begin{array}{c}\text { eCEA } \\
(\mathrm{n}=57)\end{array}$ & P-value \\
\hline NBL on diffuse-weighted MRI & $22(34.9)$ & $8(14.0)$ & $0.008^{*}$ \\
\hline Stoke/TIA & $4(6.3)$ & $3(5.3)$ & 0.800 \\
\hline Myocardial infarction & $0(0)$ & $0(0)$ & \\
\hline $\begin{array}{l}\text { Cerebral hyper-perfusion } \\
\text { syndrome }\end{array}$ & $1(1.6)$ & $0(0)$ & 0.339 \\
\hline Intracranial hemorrhage & $2(3.2)$ & $1(1.8)$ & 0.619 \\
\hline Wound complication & $2(3.2)$ & $1(1.8)$ & 0.619 \\
\hline Cranial nerve palsy & $3(4.8)$ & $1(1.8)$ & 0.359 \\
\hline Mortality & $0(0)$ & $0(0)$ & \\
\hline
\end{tabular}

Values are presented as number (\%).

cCEA, conventional carotid endarterectomy; eCEA, eversion carotid endarterectomy; NBL, new brain lesion; TIA, transient ischemic attack.

$* \mathrm{P}<0.05$, statistically significant.

Table 4. Midterm (30 days-1 year) postoperative complications

\begin{tabular}{lccc}
\hline Complication & cCEA $(\mathrm{n}=63)$ & eCEA $(\mathrm{n}=57)$ & P-value \\
Restenosis & $1(1.6)$ & $1(1.8)$ & 0.943 \\
Stoke & $1(1.6)$ & $1(1.8)$ & 0.943 \\
Mortality & $0(0)$ & $0(0)$ & \\
\hline
\end{tabular}

Values are presented as number (\%).

cCEA, conventional carotid endarterectomy; eCEA, eversion carotid endarterectomy.

Table 5. Late ( $>1$ year) postoperative complications

\begin{tabular}{lccc}
\hline Complication & cCEA $(\mathrm{n}=63)$ & eCEA $(\mathrm{n}=57)$ & P-value \\
Restenosis & $2(3.2)$ & $1(1.8)$ & 0.619 \\
Stoke & $1(1.6)$ & $0(0)$ & 0.339 \\
Mortality & $0(0)$ & $0(0)$ & \\
\hline
\end{tabular}

Values are presented as number (\%).

cCEA, conventional carotid endarterectomy; eCEA, eversion carotid endarterectomy.

no statistical differences in the early complications with the exception of a significantly higher risk for new brain lesions in the CCEA group. The incidence for new brain lesions was higher in cCEA group compared to eCEA group $(34.9 \%$ vs. $14.0 \%$, $\mathrm{P}=0.008$ ). There were no statistical differences in midterm complication between CCEA and eCEA group. Restenosis and stroke occurred in 1 of $63(1.6 \%)$ in cCEA group and 1 of 57 $(1.8 \%)$ in eCEA group $(\mathrm{P}=0.943)$. They were treated without any sequelae. And there were no deaths during the period (Table 4).

Mean follow-up duration was $29.4 \pm 23.5$ months. Over 1 year after CEA, there were 2 cases of restenosis (3.2\%) and 1 case of stroke $(1.6 \%)$ in CCEA. On the other hand, there were only 1 case of restenosis (1.8\%) and no stroke in eCEA. Although 
there was no statistical significance, the frequency of late complications was higher in the cCEA group compared to eCEA group (Table 5).

\section{DISCUSSION}

In this study, CEA was performed with acceptable periprocedural complication rates. There are no fatal complications, such as ipsilateral disabling stroke, myocardial infarction and mortality. And CCEA has a higher risk for new brain lesions within 30 days after CCEA. There were no statistical differences in midterm complication between CCEA and eCEA. In late complication, although there was no statistical significance, the frequency of restenosis and stroke was higher in the CCEA compared to eCEA.

eCEA is reported to have advantages within the procedure itself. Even though clamping times were similar in both CCEA and eCEA groups in our data, clamping times during eCEA was shorter compared with patch standard procedures $(P=0.02)$ in the EVERST trial [20]. The authors suggested that eCEA is a safe and rapid procedure. eCEA is associated with more possibility for having short operation time compared to CCEA [21]. Synthetic material is not required during eCEA and there are no problems related with synthetic materials. On the other hand, cCEA also has its own advantages. Longitudinal arteriotomy that is performed during cCEA can extend from the common carotid artery to the ICA. So, it makes for easy handling of the atherosclerotic lesions at high levels [22].

There is controversy about the surgical outcomes of these two surgical methods. The EVERST trial reported the potential superiority of eCEA. This trial was a multicenter randomized study and the first trial performed in a large population to assess the role of eCEA for treating patients with carotid artery stenosis. Preliminary results were published in 1998 [20]. Six hundred seventy-five cCEAs and 678 eCEAs were performed. The incidence of any stroke, TIA, cranial nerve injury, wound hematoma, myocardial infarction and major stroke death at 30 days after CEA was not statistically different between the two groups. It is in line with our study. New brain lesion on diffuse-weighted MRI was not investigated in the EVEREST trial. In 1999, late results of the EVEREST trial were released [23]. After 30 days of CEA, no significant differences in the neurologic outcomes, myocardial infarction and death were found between the two study groups. Restenosis occurred in 37 of $675(5.52 \%)$ in CCEA and 19 of $678(2.8 \%)$ in eCEA. In our study, restenosis during mid and late term developed in 3 of $63(4.7 \%)$ in cCEA and 2 of 57 (3.5\%) in eCEA. Although our data showed no statistical differences in restenosis rates between the two groups, late results of the EVEREST trial reported high frequency of restenosis in cCEA group compared to eCEA group. Meta-analysis by Antonopoulos et al. [24] also presented the potential superiority of eCEA. In this analysis, a total 21 studies, which consisted of 7 randomized and 14 nonrandomized studies, were analyzed. eCEA showed superiority regarding perioperative stroke, perioperative death and stroke related death in short-term outcomes, but no significant difference was observed in the risk for these outcomes between cCEA and eCEA in our study. New brain lesion on diffuse-weighted MRI was not included in the outcomes. Concerning long-term outcomes, eCEA was associated with a statistically significant reduction in coronary artery occlusion and mortality. The endarterectomy subgroups analysis from the Stent-Protected Angioplasty versus Carotid Endarterectomy (SPACE-1) trial [25] suggested that the two surgical methods have its own advantages. Demirel et al. [25] analyzed 563 patients underwent cCEA or eCEA from SPACE-1 study. Ipsilateral stroke and death within 30 days after surgery occurred more frequently in eCEA group compared to cCEA group. In our study concerning early complication, there was no statistical significance in the incidence of stroke/TIA, cCEA had a high risk for new brain lesion on diffuse-weighted MRI. cCEA was associated with a significantly higher risk of ipsilateral stroke beyond the 30 day perioperative period. There are some differences in the frequency and type of complications probably originating from regional differences, study method, patient characteristics, surgical technique and so on. But, in most studies including our research, it seems obvious that eCEA is not inferior to cCEA.

In conclusion, our data showed that eCEA was an acceptable procedure and had some advantage compared to cCEA in aspect of the early and late complication.

\section{CONFLICTS OF INTEREST}

No potential conflict of interest relevant to this article was reported.

\section{REFERENCES}

1. Sobieszczyk P, Beckman J. Carotid artery disease. Circulation 2006;114:e244-7.

2. Petty GW, Brown RD Jr, Whisnant JP, Sicks
JD, O'Fallon WM, Wiebers DO. Ischemic stroke subtypes: a population-based study of incidence and risk factors. Stroke
1999;30:2513-6.

3. Lanzino G, Rabinstein AA, Brown RD Jr. Treatment of carotid artery stenosis: 
medical therapy, surgery, or stenting? Mayo Clin Proc 2009;84:362-87.

4. Eastcott HH, Pickering GW, Rob CG. Reconstruction of internal carotid artery in a patient with intermittent attacks of hemiplegia. Lancet 1954;267:994-6.

5. Fields WS, Maslenikov V, Meyer JS, Hass WK, Remington RD, Macdonald M. Joint study of extracranial arterial occlusion. V. Progress report of prognosis following surgery or nonsurgical treatment for transient cerebral ischemic attacks and cervical carotid artery lesions. JAMA 1970; 211:1993-2003.

6. Shaw DA, Venables GS, Cartlidge NE, Bates D, Dickinson PH. Carotid endarterectomy in patients with transient cerebral ischaemia. J Neurol Sci 1984:64: 45-53.

7. Chaturvedi S, Bruno A, Feasby T, Holloway $\mathrm{R}$, Benavente $\mathrm{O}$, Cohen SN, et al. Carotid endarterectomy: an evidencebased review: report of the Therapeutics and Technology Assessment Subcommittee of the American Academy of Neurology. Neurology 2005;65:794-801.

8. Halliday A, Mansfield A, Marro J, Peto C, Peto R, Potter J, et al. Prevention of disabling and fatal strokes by successful carotid endarterectomy in patients without recent neurological symptoms: randomised controlled trial. Lancet 2004:363: 1491-502.

9. North American Symptomatic Carotid Endarterectomy Trial Collaborators. Beneficial effect of carotid endarterectomy in symptomatic patients with highgrade carotid stenosis. N Engl J Med 1991;325:445-53.

10. Ferguson GG, Eliasziw M, Barr HW, Clagett GP, Barnes RW, Wallace MC, et al. The North American Symptomatic Carotid
Endarterectomy Trial : surgical results in 1415 patients. Stroke 1999:30:1751-8.

11. Biller J, Feinberg WM, Castaldo JE, Whittemore AD, Harbaugh RE, Dempsey RJ, et al. Guidelines for carotid endarterectomy: a statement for healthcare professionals from a Special Writing Group of the Stroke Council, American Heart Association. Circulation 1998:97:501-9.

12. De Bakey ME, Crawford ES, Cooley DA, Morris GC Jr. Surgical considerations of occlusive disease of innominate, carotid, subclavian, and vertebral arteries. Ann Surg 1959;149:690-710.

13. Etheredge SN. A simple technic for carotid endarterectomy. Am J Surg 1970;120:2758.

14. Kasprzak P, Raithel D. Eversion endarterectomy of the internal carotid artery. Vasa Suppl 1992;37:83-4.

15. Shah DM, Darling RC 3rd, Chang BB, Paty PS, Kreienberg PB, Lloyd WE, et al. Carotid endarterectomy by eversion technique: its safety and durability. Ann Surg 1998;228:471-8

16. Young B, Moore WS, Robertson JT, Toole JF, Ernst CB, Cohen SN, et al. An analysis of perioperative surgical mortality and morbidity in the asymptomatic carotid atherosclerosis study. ACAS Investigators. Asymptomatic Carotid Atherosclerosis Study. Stroke 1996;27:2216-24.

17. Rothwell PM, Slattery J, Warlow CP. Clinical and angiographic predictors of stroke and death from carotid endarterectomy: systematic review. BMJ 1997; 315:1571-7.

18. Sadideen H, Taylor PR, Padayachee TS. Restenosis after carotid endarterectomy. Int J Clin Pract 2006;60:1625-30.

19. Paciaroni M, Eliasziw M, Kappelle LJ, Finan JW, Ferguson GG, Barnett HJ. Medical complications associated with carotid endarterectomy. North American Symptomatic Carotid Endarterectomy Trial (NASCET). Stroke 1999;30:1759-63.

20. Cao P, Giordano G, De Rango P, Zannetti S, Chiesa R, Coppi G, et al. A randomized study on eversion versus standard carotid endarterectomy: study design and preliminary results: the Everest Trial. J Vasc Surg 1998;27:595-605.

21. Peiper C, Nowack J, Ktenidis K, Reifenhauser W, Keresztury G, Horsch S. Eversion endarterectomy versus open thromboendarterectomy and patch plasty for the treatment of internal carotid artery stenosis. Eur J Vasc Endovasc Surg 1999;18: 339-43.

22. Connolly JE. The evolution of extracranial carotid artery surgery as seen by one surgeon over the past 40 years. Surgeon 2003;1:249-58

23. Cao P, Giordano G, De Rango P, Zannetti S, Chiesa R, Coppi G, et al. Eversion versus conventional carotid endarterectomy: late results of a prospective multicenter randomized trial. J Vasc Surg 2000;31(1 Pt 1):19-30.

24. Antonopoulos CN, Kakisis JD, Sergentanis TN, Liapis CD. Eversion versus conventional carotid endarterectomy: a metaanalysis of randomised and non-randomised studies. Eur J Vasc Endovasc Surg 2011;42:751-65.

25. Demirel S, Attigah N, Bruijnen H, Ringleb P, Eckstein HH, Fraedrich G, et al. Multicenter experience on eversion versus conventional carotid endarterectomy in symptomatic carotid artery stenosis: observations from the StentProtected Angioplasty Versus Carotid Endarterectomy (SPACE-1) trial. Stroke 2012;43:1865-71. 